14 males, $150 \mathrm{mg}$ clopidogrel per day) and the control group ( $\mathrm{n}=26$, 15 males, $75 \mathrm{mg}$ clopidogrel per day). QCA and TIMI Myocardial perfusion grading (TMPG) were used to analyse the lesion and reperfusion of the culprit vessel and myocardium. Record the information of patients in-hospital, in the 1 month and 6 months including the level of BNP, left ventricular ejection fraction (LVEF), the left ventricular peak ejection rate (LPER), the left ventricular peak filling rate (LPFR), the left ventricular time to peak ejection rate (LTPER) and left ventricular time to peak filling rate (LTPFR).

Result 1. The CTFC of the high maintenance dose group after PCI was smaller than the standard dose groupThe percentage of TMPG 3 grade was higher in the high maintenance dose group 2 . The left ventricular peak ejection rate (LPER), the left peak filling rate (LPFR) 6 months after PCI in the high maintenance dose group was higher than the control group. The left ventricular time to peak ejection rate (LTPER), left ventricular time to peak filling rate (LTPFR) 1 month after PCI in the high maintenance were lower than the control group. 3. There were less acute and subacute thrombosis cases in the high maintenance dose group than the standard dose group. There was no significant difference in haemorrhage events between two groups.

Conclusion The high maintenance dose clopidogrel can improve cardiac function. There is potential benefit in increasing coronary blood flow and improving myocardium perfusion. High maintenance dose clopidogrel decreases the acute and subacute thrombosis but do not increase the haemorrhage events.

\section{e0456 RELATIONSHIP BETWEEN HYPOKALAEMIA AT THE EARLY STAGE OF ACUTE MYOCARDIAL INFARCTION AND MALIGNANT VENTRICULAR ARRHYTHMIA}

doi:10.1136/hrt.2010.208967.456

Fu Xianghua, Oi Peng, Wang Yanbo, Wang Xuechao, Li Shiqiang, Fan Weize, Jiang Yunfa. The 2nd Hospital of Hebei Medical University, Hebei, China

Objective To investigate the relationship between hypokalaemia at the early stage of acute myocardial infarction (AMI) and malignant ventricular arrhythmia (MVA) as well as the features of hypokalaemia.

Methods Total of 302 patients were involved in this study and conformed to the following conditions: getting AMI primarily, onset was within $24 \mathrm{~h}$, accepted serum potassium test and Holter monitoring on admission, didn't use diuretics before, hyperthyroidism, diabetes, vomiting or diarrhoea resulted from gastrointestinal diseases. Relevant data including types of AMI, namely STEMI or NSTEMI; infarct sites of STEMI; time interval from onset of AMI to admission; whether or not hypokalaemia (serum potassium $\leq 3.5$ $\mathrm{mmol} / \mathrm{l}$ ) and MVA were recorded. The relationships between hypokalaemia and MVA, the time interval and hypokalaemia, types of AMI and hypokalaemia, infarct sites and hypokalaemia were analysed. SPSS13.0 was used for statistical analysis. The categorical data was processed with chi-square test and $\mathrm{p}$ values below 0.05 were considered significant.

Results The incidence of hyokalaemia for 24 patients within $3 \mathrm{~h}$ from onset of AMI to admission was $37.5 \%$. The incidence of MVA between the group with and without hypokalaemia had significant difference $(10.47 \%$ vs $3.36 \%, p<0.05)$. The incidence of hypokalaemia between the group within $3 \mathrm{~h}$ and group within $3 \mathrm{~h}$ to $24 \mathrm{~h}$ of time interval from onset of AMI to admission had significant difference $(37.5 \%$ vs $15.47 \%, \mathrm{p}<0.05)$. There was no significant difference in incidence of hypokalaemia between the group of STEMI and NSTEMI (20.35\% vs $12.68 \%, p>0.05)$. There was no significant difference in incidence of hypokalaemia between groups with anterior wall AMI and non-anterior wall AMI $(25.88 \%$ vs $18.81 \%, \mathrm{p}>0.05)$.
Conclusion At the early stage of AMI, hypokalaemia is often present. MVA was close associated with hypokalaemia at the early stage of AMI, which indicated that hypokalaemia was a cause of death.

\section{e0457 ESTABLISHMENT OF CONTRAST INDUCED NEPHROPATHY MODEL IN RATS INTERVENTION}

doi:10.1136/hrt.2010.208967.457

Fu Xianghua, Jia Xinwei, Wang Yanbo, Wang Xuechao, Zhang Jing, Fan Weize, Gu Xinshun, Jiang Yunfa. The 2nd Hospital of Hebei Medical University, Hebei, China

Objectives The purpose of this study was to establish a rat model of CIN and to evaluate its efficacy.

Methods Totally $24 \mathrm{SD}$ rats were randomly allocated into experimental group (group $\mathrm{A}, \mathrm{n}=12$ ) and control group (group $\mathrm{B}, \mathrm{n}=12$ ). After dehydration for three days, rats in group A were given intravenous MDDS, while rats in group $B$ were given intravenous normal saline (NS). Then, all rats got normal water-drinking to the end of study. Renal ultrasonic examination was performed to observe the morphologic changes, diameters of renal artery and blood flow in renal artery. Blood samples were taken to measure the level of serum creatinine. The tissue of kidney were incised for microscope and electron microscope study.

Results The dimensions of the two groups before and after dehydration were not different. It gradually enlarged after CM injection. These changes were the most obvious at 6 and $12 \mathrm{~h}$, which did not recover at $24 \mathrm{~h}$. The PSV, EDV, S/D and VTI were lowerest at $6 \mathrm{~h}$ and then recover to normal level at $24 \mathrm{~h}$. RI was increased after CM injection, the lowest occurred at $6 \mathrm{~h}$, and recovered to normal level at $24 \mathrm{~h}$. Serum creatinine was significantly elevated after dehydration, the highest level occurred at $12 \mathrm{~h}$ and then began to recover at $24 \mathrm{~h}$. Microscope examination to renal sample at $12 \mathrm{~h}$ found patch disappearance of tubular structure, widely congestion at medullar area. No pathological glomerular changes were found under microscope. Electron microscope examination found desquamation, sparseness of microvillous of tubular endothelium, membrane confusion, disappearance, swelling, fragmentation of the MIT, with obstrcted tubular lumen and basal membrane swelling.

Conclusion Combined with dehydration, intravenous injection of contrast lead to obvious acute kidney injury, with the changes of kidney tissue pathology, haemodynamics and kidney functions which are similar to the characteristics of CIN in humanbeings.

\section{e0458 COMPARATIVE STUDY OF ASPIRIN AND CLOPIDOGREL IN HIGH RISK ACS}

doi:10.1136/hrt.2010.208967.458

${ }^{1}$ Ren Yihong, ${ }^{1}$ Chen Yundai, ${ }^{1} Z$ Zhao Ming, ${ }^{2}$ Chen Jinsong, ${ }^{1}$ Chen Lian. ${ }^{1} P L A$ General Hospital/Cardiovascular Department, ${ }^{2}$ PLA General Hospitalclinical Laboratory

Background It is not reasonable to administrate the same dosage of antiplatelet medicine to all patients with ACS regardless of patients' height, weight, metabolism and effectiveness of those medicines. And thromboelastography (TEG) has provided a relatively stable, convenient, duplicable method for testing activity of platelet in recent years.

Objective To investigate the inhibition levels and characteristics of frequency distributions of platelet aggregation after antiplatelet therapy with aspirin and clopidogrel in Chinese patients with ACS undergoing PCI.

Methods High risk Patients with ACS received PCI after administration of loading dosage of clopidogrel $(600 \mathrm{mg})$ and aspirin (300 mg) subsequence with maintenance dose of clopidogrel $(75 \mathrm{mg}$ per day) and aspirin (100 mg per day) for one year. Blood sample was gotten $24-48 \mathrm{~h}$ after PCI for the test of TEG-mapping in order to 